\title{
f ACTORES DE RESILIENCIA qUE INCIDEN EN EL ÉxITO ACADÉMICO DE ESTUDIANTES EN LA MODALIDAD DE EDUCACIóN A DISTANCIA ${ }^{36}$
}

\author{
Diana Patricia Landazábal Cuervo, ${ }^{37}$ María del Pilar Cardona, ${ }^{38}$ \\ Maria Yasbleidy Ruiz Manzanares ${ }^{39}$
}

\begin{abstract}
RESUMEN
En la Educación a Distancia se presentan dos tipos de fenómenos; el primero, tiene que ver con el alto grado de estudiantes que desertan durante su proceso de formación, $50 \%$ según investigaciones realizados por el Ministerio de Educación Nacional (MEN); y el segundo, está relacionado con los estudiantes que a pesar de las situaciones adversas a las que se enfrentan (en lo personal y en lo educativo), tienen éxito académico durante su proceso de aprendizaje y formación. Estos fenómenos han llamado la atención de los investigadores, y se han venido estudiando desde la resiliencia. En la investigación eje de este artículo, se estudiaron los factores resilientes relacionados con la permanencia y el éxito académico de los estudiantes de un curso de psicología en la Universidad Nacional Abierta y a Distancia (UNAD). Se aplicó la prueba CREU, y una entrevista semiestructurada. Los resultados mostraron que los grupos colaborativos fomentan la resiliencia comunitaria ya que el interactuar con otros promueve el aprendizaje social, potencializando así las fortalezas propias y la de los demás; los estudiantes que reconocen sus debilidades y falencias y buscan la forma de corregirlas, por lo general poseen el factor de la introspección, que contribuye al éxito académico. Para próximas investigaciones en este tema es importante tener en cuenta variables como: sexo, edad, semestre, estado civil, etc., para analizar si estas variables inciden en el rendimiento académico de los (as) estudiantes de esta modalidad.
\end{abstract}

Palabras clave: éxito académico, resiliencia, educación a distancia, deserción, trabajo en equipo, iniciativa.

\begin{abstract}
Distance education exhibits two phenomena: first, the high percentage of students who drop out during their formative process: $50 \%$, according to research by the National Education Ministry (NEM)1; second, that of students who, despite adverse conditions (in their personal life or their education), achieve academic success during their learning and formative processes. These phenomena have attracted the attention of researchers who have studied

36 Esta es una investigación como opción de grado de las estudiantes Audrey Claro Ortega, Maria Yasbleidy Ruíz y María del Pilar Cardona, del Programa de Psicología Social Comunitaria de la UNAD.

37 Docente e Investigadora de la UNAD. Correo: dlandaz1227@yahoo.com

38 Psicóloga de la UnAD. Correo: pili2814@gmail.com

39 Psicóloga de la UNAD. Correo: yasbleidyr@gmail.com
\end{abstract}


them from a perspective of resilience. The study described in this article focused on resilience as a factors of the continuance and academic success of students of a Psychology course at the National Open University for Distance Education (UNAD - Universidad Nacional Abierta y a Distancia). A CREU2 test was applied and a semi-structured interview was conducted. The results show that collaborative groups foster community resilience because interaction with other groups promotes social learning, and therefore brings out their own strengths as well as those of the others. In general, students who acknowledge their weaknesses and failings, and seek to correct them, possess a level of introspection, which is a factor for academic success. In future research on this subject, it is important to consider other variables like genre, age, semester, marital status, etc., and to analyze their impact on academic performance in distance education.

Key words: academic success, resilience, distance education, desertion, teamwork, initiative.

Recibido: 21 de mayo de 2009 Aceptado: 23 de octubre de 2009

\section{INTRODUCCIóN}

La Universidad Nacional Abierta y a Distancia UNAD se encuentra dividida en ocho zonas a lo largo de la geografía nacional, ocho zonas: Amazonia- Orinoquia, Caribe, Centro Oriente, Centro, Centro Boyacá, Centro Sur, Occidente y Sur. Cuenta con un total de 55 CEADs (Centros de Educación Abierta y a Distancia) y 17 CERES (Centros Regionales de Educación Superior). En la actualidad, la UNAD cuenta con más de cincuenta mil estudiantes, de los cuales veintinueve mil hacen parte de los estratos 1 y 2 , en todo el territorio nacional.

Una de las mayores problemáticas de las instituciones de educación superior en la modalidad presencial o a distancia, es la deserción. En la UNAD, se han venido desarrollando algunas investigaciones con el objeto de comprender este fenómeno y por tanto, encontrar estrategias que posibiliten aumentar la retención y el éxito académicos de los estudiantes.

Por ello, el Sistema Nacional de Consejería realizó una investigación sobre el perfil del estudiante Unadista (2008), en el que se caracterizó la población estudiantil con el fin de partir de los resultados, para definir estrategias metodológicas, pedagógicas, académicas y didácticas, que contribuyeran a mejorar la retención de los estudiantes, el rendimiento académico y el tránsito exitoso por el sistema de educación superior en el modelo de educación que ofrece la UNAD. Los resultados arrojados por la investigación demuestran que el $94 \%$ de los estudiantes pertenece a los estratos socioeconómicos 0 al 3; 28\% de las estudiantes son madres cabezas de hogar; el 73\% de la población estudiantil está vinculado laboralmente, además, del $70 \%$ de la población que se ha retirado temporalmente de la universidad por razones laborales y económicas.

Otros antecedentes de investigaciones en Educación a Distancia como la de Arguedas (2004), que se realizó en la unED de Costa Rica, acerca de la potenciación de la resiliencia como alternativa para la permanencia y éxito académico de los estudiantes en dicha unirversidad, determinó que la potenciación y promoción de la resiliencia es una alternativa pertinente que se puede trabajar con la comunidad estudiantil. 
Peralta S., (2006), realizó una investigación sobre factores resilientes asociados al rendimiento académico en la Universidad del Sucre (Colombia). En su estudio, describió las características más relevantes que poseen los estudiantes con alto y bajo rendimiento, se comparon los grupos, con el fin de conocer si existían diferencias entre los factores resilientes y se identificó cuáles de las variables pueden influir directamente en los resultados de los estudiantes. Se encontró lo siguiente: 1: Que los estudiantes con mejores notas presentan los factores de introspección, interacción, iniciativa, independencia, moralidad y pensamiento crítico. 2. El factor Iniciativa, es el único entre todos los demás factores resilientes, que es determinante en el rendimiento académico alto o bajo de los estudiantes en esta Universidad.

Otros factores que tienen que ver con el éxito académico son los que están asociados con las características del estudiante como son, sexo, edad, estado civil; el entorno familiar, la escuela en la que realizó los estudios del nivel secundario, la situación laboral (si trabaja o no; la cantidad de horas de trabajo) y la carrera que cursa (Di Gresia, Porto y Ripani, 2002).

(Schiefelbein y Simmons, (1981) comprobaron los resultados de otros autores que dicen que la planificación y dedicación adecuada del tiempo de estudio se relacionan significativamente con el rendimiento académico. El bajo rendimiento se encuentra entre los estudiantes que cursan tercer, cuarto y quinto semestre debido a que existe mayor número de estudiantes y la exigencia es mayor.

A partir de estos antecedentes, en la investigación que se presenta en este artículo se consideró propicio estudiar la resiliencia en estudiantes de la modalidad de Educación a Distancia y analizar cuáles son los factores de la resiliencia que tienen los estudiantes que presentan éxito académico en el área Temática de Seminario Regional I, II semestre de 2007. Para ello se identificaron los factores resilientes de los estudiantes del curso y los factores resilientes que influyen en el éxito académico de éstos estudiantes.

\section{El estudio de la resiliencia en los contextos educativos}

La resiliencia se ha convertido en una herramienta de intervención en el campo educativo o en los diversos aspectos del aprendizaje, debido a que el estudio se puede ver como un factor protector haciendo alusión a la perseverancia que busca la superación personal de los individuos. Por su parte, Werner, (1990), citado por Peralta S., (2006), en sus investigaciones hizo una relación positiva entre la presencia de factores protectores y el buen desempeño de los niños en la escuela. Esto deja ver que pueden existir relaciones significativas entre la capacidad resiliente y el rendimiento académico de los estudiantes, entendiendo rendimiento como una variable multidimensional influenciada por factores familiares, sociales, pedagógicos y por las características del propio sujeto. Éstas se organizan en tres categorías: procesos escolares, atributos del docente y rasgos personales del sujeto que aprende y que son determinantes del rendimiento, Schiefelbein, (1982).

La resiliencia es un proceso fundamentalmente adaptativo que pretende aportar a la calidad de vida de las personas. Está fundamentada en el desarrollo de la actitud (incluyendo 
sentimientos, pensamientos y comportamientos) de recuperación y adecuación que se desarrolla ante las situaciones a las cuales las personas pueden verse enfrentadas.

En los entornos educativos, la resiliencia busca construir ambientes propicios para que las personas puedan desarrollar todo su potencial, generando así capacidades y factores que incidan en la permanencia y en el desempeño académico durante el proceso educativo. Instituciones de educación superior bajo modelos a distancia enfrentan el reto de lograr generar un ambiente educativo que propicie la permanencia y éxito académico de sus estudiantes bajo situaciones complejas dadas las exigencias personales, académicas y pedagógicas que la metodología pide.

Entendiendo los conceptos de resiliencia y rendimiento, se podría decir entonces, que posiblemente los estudiantes que tienen mejor desempeño académico poseen mayor número de factores resilientes. Esto se debe a que estas personas son capaces de afrontar satisfactoriamente las amenazas y retos de la vida universitaria. En cambio, las personas de bajo rendimiento académico poseen menos presencia de factores protectores, es decir, que se encuentran en riesgo académico. Concibiendo el riesgo como las características o cualidades de una persona o comunidad asociadas a una elevada probabilidad de daño biopsicosocial Kotliarenco, et. al., (1998).

\section{METODOLOGÍA}

\section{Enfoque}

El enfoque de la investigación es cualitativo y cuantitativo. Se realizaron entrevistas, una clasificación de la información y una categorización de la misma. Se realizó la aplicación del cuestionario CREU y se realizó un análisis estadístico, para medir los factores de la resiliencia y encontrar cuál es el factor que incide más en el éxito académico de la muestra.

\section{Tipo de investigación}

La investigación es de tipo descriptivo, ya que busca realizar una descripción de la incidencia de los factores resilientes en el éxito académico, de los estudiantes integrados en un programa de educación a Distancia.

\section{Población y muestra}

El universo poblacional es de 63 estudiantes, matriculados en el Curso Seminario Regional I, del segundo semestre del 2007, del programa de Psicología de la UNAD en Bogotá, que tuvieron éxito académico durante el semestre.

\section{Técnicas}

\section{Entrevista semiestructurada}

En la entrevista se evaluó: (1) los factores de permanencia que los estudiantes tienen para no desertar de la UNAD, (2) los factores de deserción por los cuales a los estudiantes les gustaría 
abandonar el proceso académico en la UNAD y (3) la percepción que tienen los estudiantes de las diferentes estrategias metodologías utilizadas en la UNAD para la adquisición de los conocimientos y su relación con el éxito o la deserción.

\section{Cuestionario de resiliencia para estudiantes universitarios, CREU}

Se aplicó el cuestionario CREU. El instrumento es un cuestionario que sirve para medir los factores resilientes en estudiantes universitarios. Éste posee una validez de constructo basada en la teoría de los autores Emmy Werner (1982) y Wolin y Wolin (1993), que se centra en explicar los factores personales que sirven de protección y fuerza a las personas en situaciones difíciles o adversas. Los factores que se tienen en cuenta en la aplicación de la prueba son: introspección, iniciativa, humor, interacción, independencia, creatividad, moralidad y pensamiento crítico, Peralta S., (2006).

\section{Procedimiento}

Con base en la lista de estudiantes matriculados en el curso académico Seminario Regional, se verificó que 63 estudiantes presentaban éxito académico con nota final superior a 4.2. Se seleccionó de manera aleatoria una muestra representativa del $20 \%$, para el posterior análisis de los factores resilientes.

Las entrevistas y cuestionarios se realizaron en las instalaciones de la Universidad, en la sede José Celestino Mutis, se contactó a los estudiantes de manera personal y se les explicó el objetivo de la investigación, se pidió su participación y colaboración para la posterior aplicación de las pruebas, algunos de ellos presentaron la entrevista de manera personal y el cuestionario lo enviaron por medio del correo electrónico a las investigadoras. El proceso duró aproximadamente tres meses debido a la modalidad de estudio de la Universidad. Esta aplicación se efectuó en el primer semestre del 2008, a los estudiantes seleccionados.

Se hizo un análisis estadístico descriptivo y de análisis de contenido de las entrevistas. Esto permitió alcanzar los objetivos de la investigación y reconocer los factores de resiliencia de los estudiantes que presentaron éxito académico.

\section{RESULTADOS}

\section{Entrevistas}

En la tabla 1 se presentan las categorías y subcategorías que surgieron a partir de las entrevistas con sus respectivas definiciones. Con base en ellas se realizaron los cuadros categoriales que se presentan en las figuras 1 y 2. 
Tabla 1. Definición de categorías y subcategorías

\begin{tabular}{|c|c|c|c|}
\hline CATEGORÍAS & $\begin{array}{l}\text { SUBCATEGORÍAS DE } \\
\text { PRIMER NIVEL }\end{array}$ & $\begin{array}{l}\text { SUBCATEGORÍAS DE } \\
\text { SEGUNDO NIVEL }\end{array}$ & $\begin{array}{l}\text { SUBCATEGORÍAS } \\
\text { DE TERCER NIVEL }\end{array}$ \\
\hline \multirow{7}{*}{$\begin{array}{l}\quad \text { 1. f ACTORES DE } \\
\text { PERMANENCIA } \\
\text { Definición: Hace referencia a } \\
\text { los motivos por los cuales los } \\
\text { estudiantes desean continuar } \\
\text { y han permanecido en el pro- } \\
\text { grama de Psicología de una } \\
\text { manera constante. }\end{array}$} & 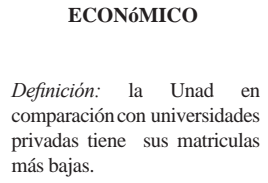 & \begin{tabular}{l}
\multicolumn{1}{c}{ BAJOS COSTOS } \\
EN LA MATRÍCULA \\
Definición: se refiere al bajo costo \\
que se debe cancelar por concep- \\
to de matrículas en comparación \\
con las universidades privadas.
\end{tabular} & \\
\hline & $\begin{array}{l}\quad \text { MODALIDAD DE } \\
\quad \text { ESTUDIO } \\
\text { Definición: hace referencia a } \\
\text { la metodología implementada } \\
\text { en la universidad, es decir a } \\
\text { Distancia }\end{array}$ & 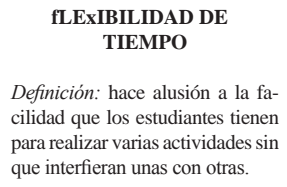 & $\begin{array}{l}\text { PARA EL TRABAJO } \\
\text { Definición: hace alusión al } \\
\text { tiempo que tienen para estudiar } \\
\text { sin interferir en las actividades } \\
\text { laborales. }\end{array}$ \\
\hline & & & $\begin{array}{c}\text { PARA LA } \\
\text { ORGANIZACIóN } \\
\text { DE OTRAS } \\
\text { ACTIVIDADES } \\
\text { Definición: hace la referencia } \\
\text { al tiempo que se tienen para } \\
\text { realizar varias actividades sin } \\
\text { que interfieran unas con otras. }\end{array}$ \\
\hline & & \begin{tabular}{l}
\multicolumn{1}{c}{ ESTRATEGÍAS } \\
DE APRENDIZAJE \\
Definición: hace referencia a las \\
herramientas metodologías uti- \\
lizadas en la uNAD para la adqui- \\
sición del conocimiento.
\end{tabular} & $\begin{array}{l}\text { GRUPALES } \\
\text { Definición: son espacios ofre- } \\
\text { cidos por la Universidad para } \\
\text { aclarar dudas, socializar lo } \\
\text { aprendido y ampliar los co- } \\
\text { nocimientos sobre un tema } \\
\text { específico. }\end{array}$ \\
\hline & & & $\begin{array}{l}\text { INDIVIDUALES } \\
\text { Definición: espacios dados } \\
\text { por los tutores para aclarar } \\
\text { dudas y brindar asesorías en } \\
\text { los trabajos. }\end{array}$ \\
\hline & & & \begin{tabular}{l}
\multicolumn{1}{c}{ APRENDIZAJE } \\
AUTóNOMO \\
Definición: hace alusión a la \\
responsabilidad que asume \\
cada estudiante con su apren- \\
dizaje, el cual repercute en su \\
formación profesional.
\end{tabular} \\
\hline & & & $\begin{array}{l}\text { GRUPOS } \\
\text { COLABORATIVOS } \\
\text { Definición: son grupos } \\
\text { integrados por máximo cinco } \\
\text { estudiantes cuyo objetivo es } \\
\text { trabajar en pro de unas metas } \\
\text { conjuntas en torno a una } \\
\text { actividad académica }\end{array}$ \\
\hline
\end{tabular}




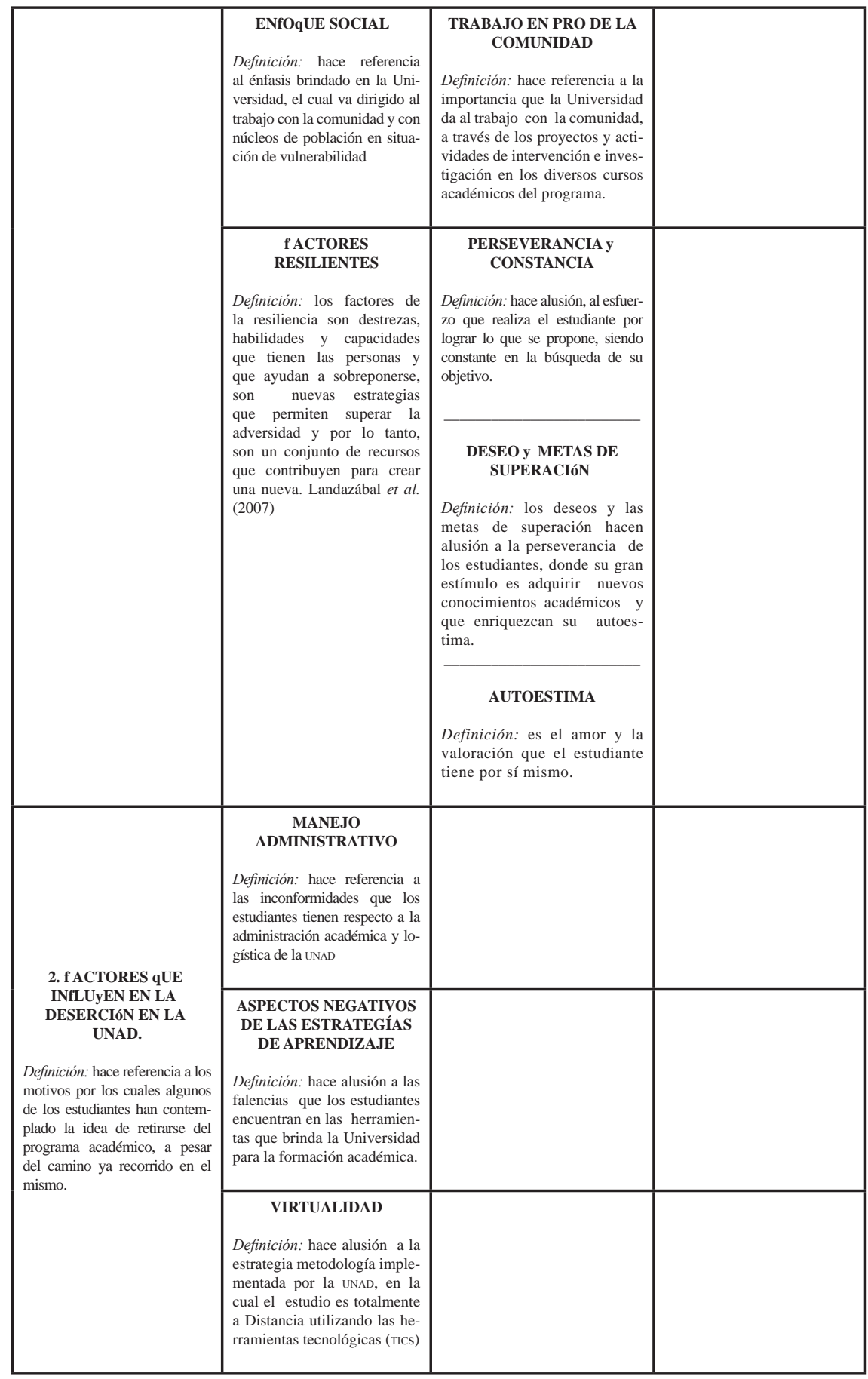




\begin{tabular}{|c|c|c|}
\hline \multirow{2}{*}{$\begin{array}{l}\text { 3. PERCEPCIóN DE } \\
\text { LOS ESTUDIANTES } \\
\text { SOBRE LA fORMACIóN } \\
\text { PROOESIONAL } \\
\text { EN LA UNAD. } \\
\text { Definición: Hace referencia al } \\
\text { concepto que los estudiantes } \\
\text { se han creado, acerca de la } \\
\text { formación y la calidad de la } \\
\text { misma. }\end{array}$} & $\begin{array}{l}\text { VENTAJAS } \\
\text { Definición: hace referencia } \\
\text { a las fortalezas que los estu- } \\
\text { diantes encuentran en la for- } \\
\text { mación ofrecida por la UNAD } \\
\text { en el programa de Psicología. }\end{array}$ & $\begin{array}{l}\text { fORMACIóN } \\
\text { PSICOSOCIAL } \\
\text { Definición: hace referencia al } \\
\text { gran interés que los estudiantes } \\
\text { le encuentran a la formación y a } \\
\text { la pedagogía que implementa la } \\
\text { UNAD en el campo psicosocial. }\end{array}$ \\
\hline & \begin{tabular}{l}
\multicolumn{1}{c}{ DESVENTAJAS } \\
Definición: hacen referencia a \\
las dificultades e inconformi- \\
dades de los estudiantes en el \\
currículo académico.
\end{tabular} & $\begin{array}{l}\text { COMPLEMENTACIóN } \\
\text { DEL PROGRAMA } \\
\text { Definición: hace referencia } \\
\text { a la desventaja que los } \\
\text { estudiantes le ven al currículo } \\
\text { de psicología, debido a que } \\
\text { baja su competitividad laboral } \\
\text { comparado con egresados de } \\
\text { otras universidades. }\end{array}$ \\
\hline
\end{tabular}

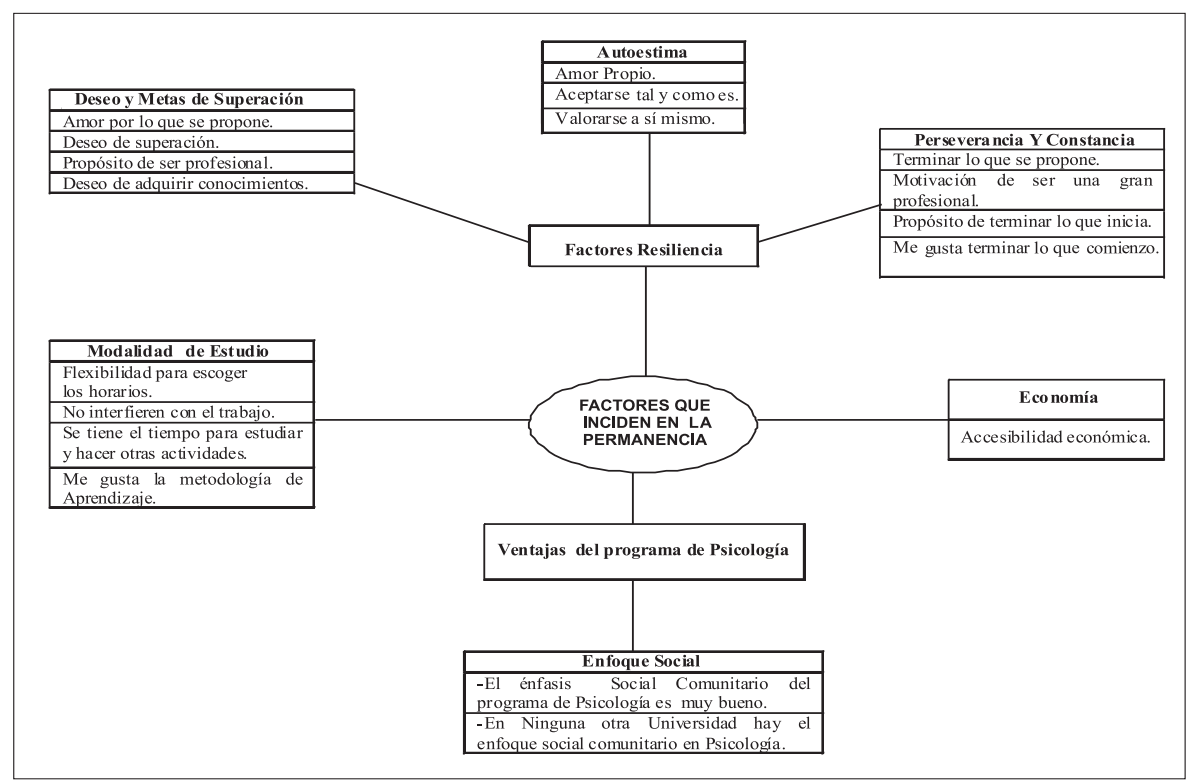

figura 1. Factores de permanencia que los estudiantes manifiestan los han mantenido en su proceso académico en la UNAD

En este esquema, se puede evidenciar los diferentes factores que inciden en la permanencia de los estudiantes en la UNAD. En lo referente a los factores de la resiliencia se encuentran las metas y deseos de superación, autoestima y la persistencia. Otros de los factores que repercuten de manera positiva en la permanencia son: la modalidad de estudio porque se adapta a las necesidades de la población de estudio y la accesibilidad económica ya que la mayoría de la población pertenece al nivel socioeconómico 0 al 3. Otro factor importante en la permanencia ha sido el énfasis social comunitario, ya que permite intervenir diferentes problemáticas sociales. 


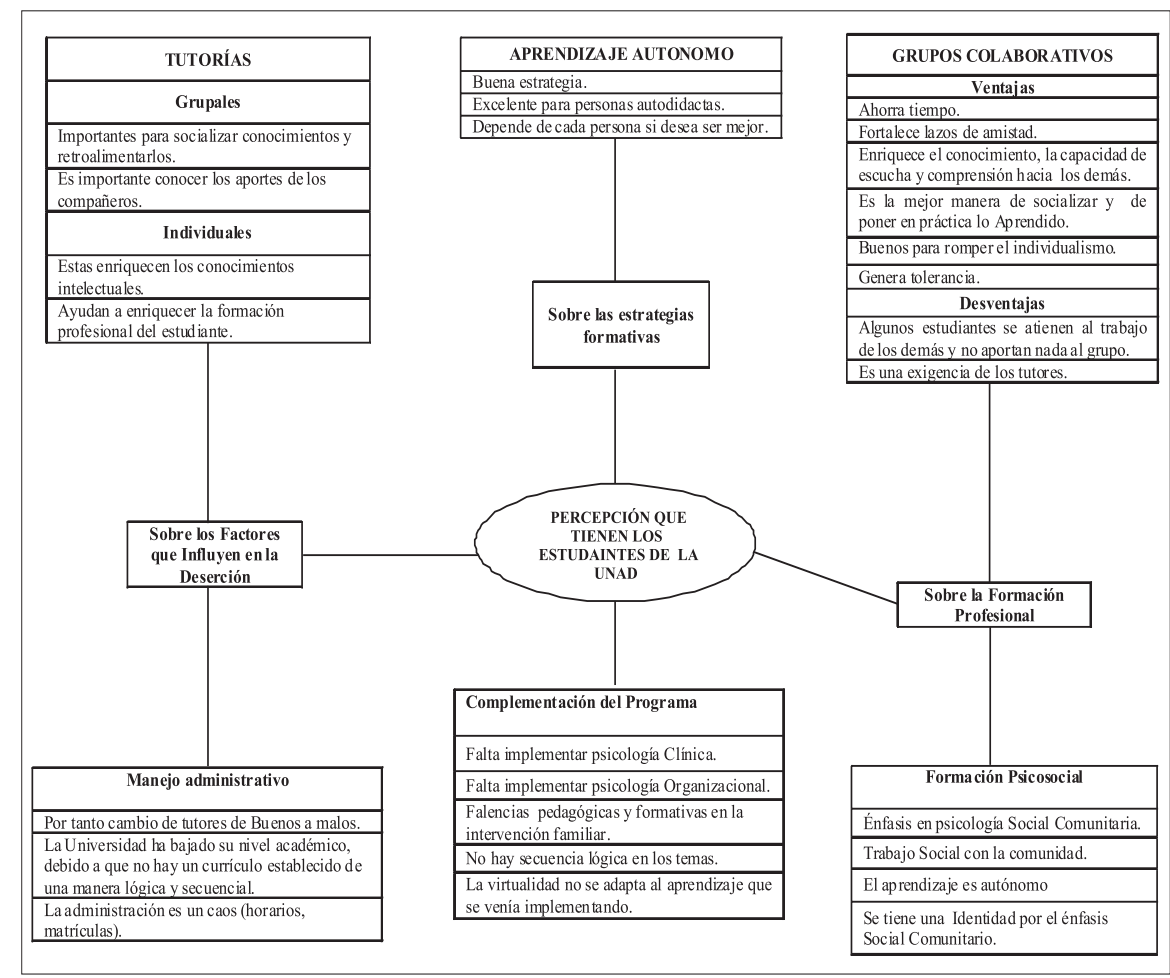

figura 2. Respuestas dadas por los estudiantes respecto a la percepción que tienen frente de la UNAD

En este cuadro categorial se puede evidenciar el concepto que tienen los estudiantes acerca de la Universidad. Frente a aspectos como la formación profesional, las estrategias formativas, las tutorías (individuales, grupales), los grupos colaborativos y el aprendizaje autónomo, además de los factores que influyen a la hora de contemplar una posible deserción.

Los estudiantes manifiestan que uno de los principales motivos por los que desertan es por la mala organización administrativa, que repercute en los horarios de tutorías que se establecen en el inicio del semestre, ya que en varias ocasiones cambian el horario una y otra vez sin tener en cuenta las dificultades que estos tienen para asistir, además, manifiestan que muchas veces, al terminar el semestre, no les aparecen registradas las notas. Otro factor es el incremento que se hace a la matrícula y a los créditos académicos semestralmente ya que a la universidad se le está olvidando que la mayoría de estudiantes son de estratos 1 y 2 y que carecen de recursos económicos para cubrir costos elevados.

\section{Cuestinario CREU}

A continuación se presentan los resultados del Cuestionario de resiliencia para estudiantes universitarios, CREU. 
Tabla 2. Tabla general del nivel de frecuencia de factores resilientes manifestados en los estudiantes que contestaron el cuestionario.

\begin{tabular}{|l|r|r|r|r|r|r|}
\hline \multicolumn{7}{|c|}{ fACTORES RESILIENTES/ NIVEL DE fRECUENCIA } \\
\hline Enunciados & $\begin{array}{c}\mathbf{1} \\
\text { Nunca }\end{array}$ & $\begin{array}{c}\text { 2 Casi } \\
\text { nunca }\end{array}$ & $\begin{array}{c}\text { 3 A } \\
\text { veces }\end{array}$ & $\begin{array}{c}\text { 4 Por lo } \\
\text { general }\end{array}$ & $\begin{array}{c}\mathbf{5} \\
\text { Siempre }\end{array}$ & frecuencia \\
\hline Introspección & 15 & 14 & 31 & 45 & 40 & 145 \\
\hline Interacción & 8 & 6 & 21 & 50 & 34 & 119 \\
\hline Iniciativa & 13 & 22 & 39 & 38 & 24 & 136 \\
\hline Interdependencia & 17 & 13 & 22 & 31 & 22 & 105 \\
\hline Humor & 7 & 5 & 23 & 17 & 11 & 63 \\
\hline Creatividad & 9 & 12 & 32 & 28 & 15 & 96 \\
\hline Moralidad & 10 & 15 & 27 & 31 & 24 & 107 \\
\hline Pensamiento Crítico & 7 & 8 & 14 & 36 & 32 & 97 \\
\hline
\end{tabular}

En la anterior tabla se puede evidenciar el nivel de frecuencia con la que se presentan cada uno de los factores de resiliencia, encontrándose que los factores de introspección, la iniciativa y la interacción por lo general están presentes en los estudiantes que manifiestan éxito académico.

\section{factor de la introspección}

El 31.0\% de los estudiantes manifiesta que por lo general presentan el factor resiliente, es decir que son capaces de reconocer sus fortalezas, capacidades y debilidades, comprender a los otros, reflexionar sobre su propia experiencia y aprender de sus errores. Seguida del $28.0 \%$ que dice siempre presentar el factor, posteriormente hay un $21.0 \%$ que expresa sólo a veces presentar el factor, un $10.0 \%$ manifiesta casi nunca presentar el factor $\mathrm{y}$, por último, el 10.0\% manifiesta nunca presentar el factor resiliente.

\section{Estudiantes}

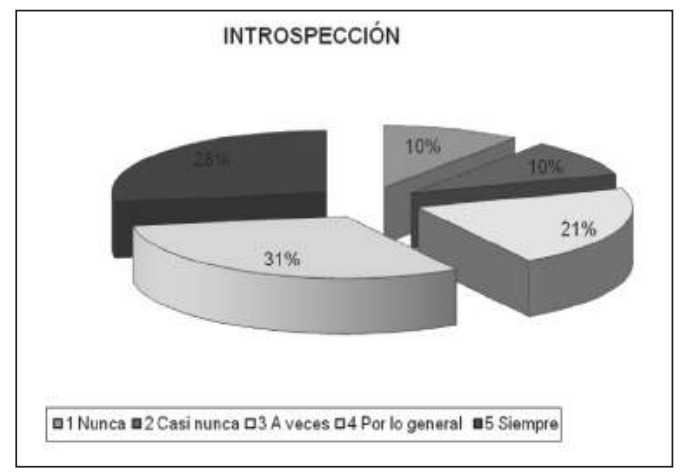

figura 3. Introspección 


\section{factor de la interacción}

El 37.0\%, de los estudiantes por lo general presenta éste factor resiliente, es decir, que posee un respaldo emocional de otras personas, son personas seguras de sí mismas, solidarias y generan relaciones gratificantes, se interesan por el bienestar propio y de los demás. Seguido de un $33.0 \%$ que manifiesta poseer siempre el factor resiliente, posteriormente existe un $15 \%$ que dice sólo a veces presentar el factor resiliente, seguido de un $8.0 \%$ que manifiesta casi nunca tener el factor y finalmente un $7.0 \%$ que expresa siempre poseer el factor de la resiliencia.

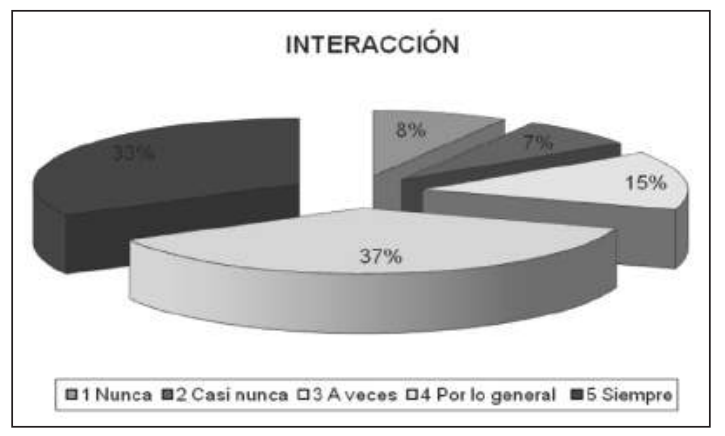

figura 4. Nivel de frecuencia del factor de la interacción presentado en los estudiantes

\section{factor de la iniciativa}

El 42.0\% de los estudiantes encuestados manifiesta que son personas motivadas por alcanzar sus propios objetivos y tienen cierta independencia emocional de las personas que quieren, son personas autónomas y perseverantes. Luego sigue el $28.0 \%$ de estudiantes que dice siempre presentar el factor, posteriormente, está el $18.0 \%$ de los estudiantes que dice que a veces y un $7.0 \%$ que dice siempre presentar el factor y por último un $5.0 \%$ expresan que nunca evidencian este factor resiliente.

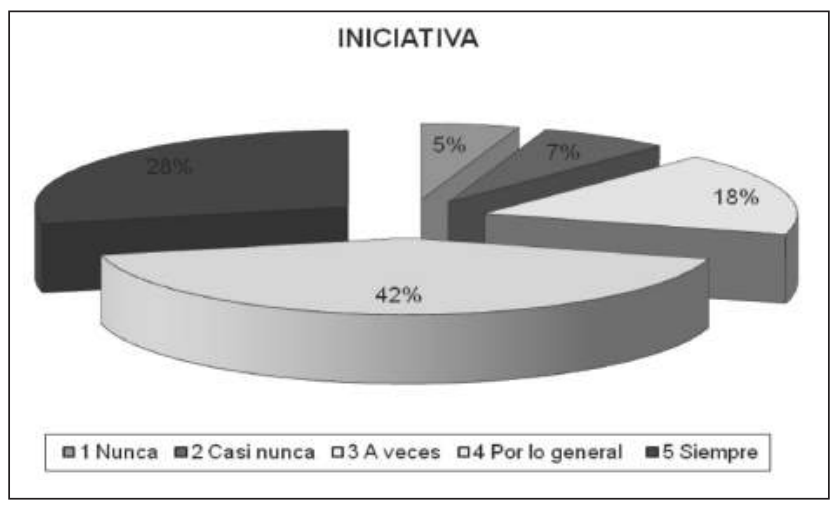

figura 5. Nivel de frecuencia del factor de Iniciativa presente en los estudiantes 


\section{factor de la interdependencia}

El 30.0\% de los estudiantes encuestados expresa que por lo general tienen este factor, es decir, que ocasionalmente las personas presentan comportamientos dirigidos hacia una meta, tienen una inclinación por el estudio, participan de actividades extracurriculares y poseen características de líderes.

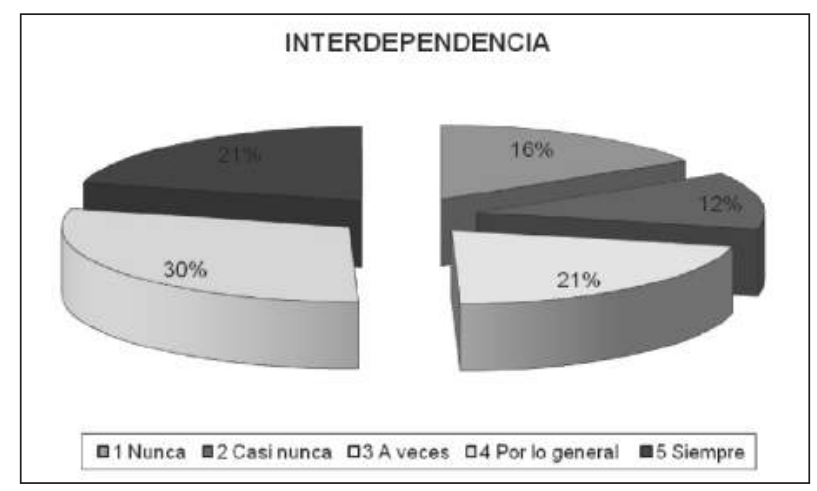

figura 6. Nivel de frecuencia del factor de la interdependencia presente en los estudiantes

\section{factor del sentido del humor}

El 37.0\% de los estudiantes encuestados manifiestan que a veces presenta el factor resiliente; el sentido del humor, hace parte de una actitud positiva que tienen los seres humanos para afrontar las experiencias vividas, además evidencia la confianza que el individuo tiene de sí mismo Luego sigue un $22.0 \%$ que manifiesta que por lo general presenta este factor, posteriormente un $27.0 \%$ dice que por lo general presentan el factor, luego viene $17.0 \%$ que expresa siempre haber tenido el factor, lo que significa que estos son capaces de conservar el sentido del humor en situaciones difíciles y, por lo tanto, pueden minimizar los hechos negativos, mediante la ridiculización o el chiste.

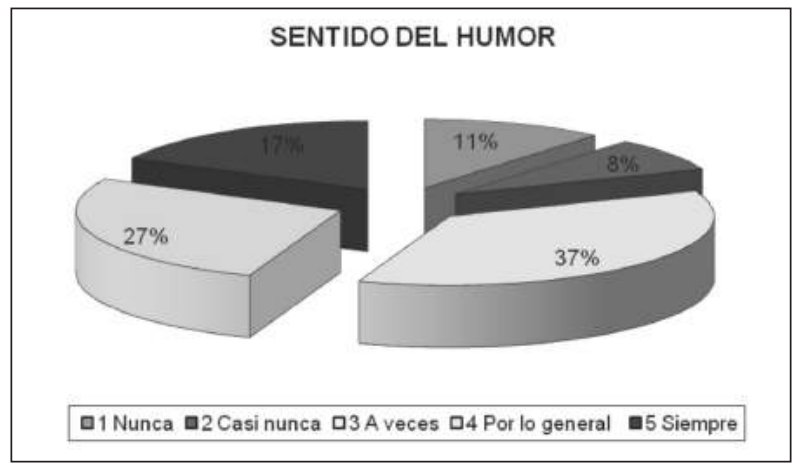

figura 7. Nivel de frecuencia del factor del sentido del humor presente en los estudiantes 


\section{factor de la creatividad}

El 33.0\% de los estudiantes encuestados manifiesta sólo a veces presentar el factor, las personas que poseen este factor, son aquellas que integran el pensamiento con la imaginación y les gusta la estética, el $29.0 \%$ dice que por lo general lo presentan, lo que quiere decir que se dedican al desarrollo de habilidades artísticas, se interesan por modificar su entorno, les gusta enfrentase al cambio y son de pensamiento divergente, es decir que se dedican al desarrollo de habilidades artísticas, se interesan por modificar su entorno, les gusta enfrentase al cambio y son de pensamiento divergente, Peralta S., y otros (2006). Luego sigue un 16.0\% de estudiantes que dice presentar el factor, un $13.0 \%$ otro tanto que expresa que casi nunca presentan el factor y finalmente un $9.0 \%$ dice que nunca presentan el factor.

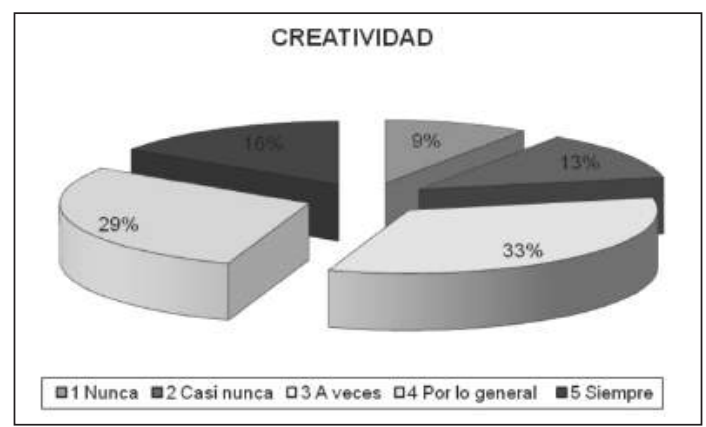

figura 8. Nivel de frecuencia del factor de la creatividad presente en los estudiantes

\section{factor de la moralidad}

El 29.0\% de los estudiantes encuestados dice que por lo general presenta el factor resiliente, lo que significa que estos se dedican a enriquecer y desarrollar sus valores, identifican las situaciones que favorecen el bien, poseen la capacidad de servicio y pueden sacrificar el placer inmediato para obtener satisfacciones a largo plazo, Peralta S., y otros (2006). Luego sigue del $25.0 \%$ que manifiesta sólo a veces presentar el factor, un $23.0 \%$ que manifiesta siempre presentarlo, luego esta un $14.0 \%$ que expresa que casi nunca tiene el factor, y finalmente el restante $9.0 \%$ expresan que nunca lo manifiestan.

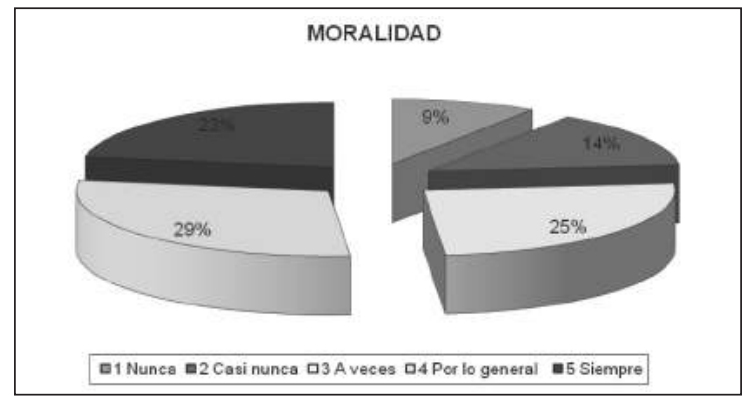

figura 9. Nivel de frecuencia del factor de moralidad presente el los estudiantes 


\section{factor pensamiento crítico}

El $29.0 \%$ de los encuestados manifiesta que, a veces, presenta este factor resiliente, en los momentos en los cuales los seres humanos actúan con la razón, hacen lo que deben hacer así no sea lo que quieren.

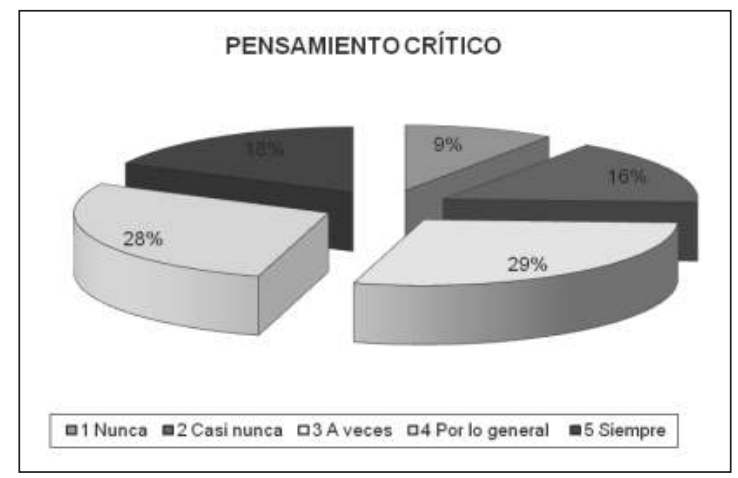

figura 9. Nivel de frecuencia del factor de pensamiento crítico presente en los estudiantes

\section{DISCUSIóN}

En esta investigación se encontró que la resiliencia juega un papel importante en el proceso educativo como lo afirma Grotberg E., (1998). Es un proceso que va más allá de una respuesta a una adversidad e incorpora tres aspectos: el primero consiste en la promoción de factores resilientes al maximizar el potencial de las personas para salir adelante, desarrollarse y crecer; el segundo es el compromiso con el comportamiento resiliente en el cual se da una interacción entre los distintos factores de la resiliencia que permiten identificar la adversidad, buscar opciones, tomar decisiones y encontrar las respuestas más convenientes; finalmente, una valoración de los resultados desde la perspectiva de la resiliencia, no sólo en términos de cómo se logró enfrentar la situación, sino también para beneficiarse y aprender de ella, mejorando la calidad de vida propia y de los otros.

Frente al primer aspecto se puede reafirmar que los estudiantes de la población muestra se consideran personas con éxito académico porque poseen factores resilientes tales como la perseverancia, la iniciativa y la introspección lo que indica que son estudiantes con una metas claras, que luchan por alcanzar sus objetivos y que su deseo es ser mejores cada día, sin dejar de lado, que reconocen sus fortalezas y debilidades y que asumen responsablemente sus actividades académicas.

En el segundo aspecto se puede evidenciar en las respuestas dadas por los(as) estudiantes, que a pesar de las adversidades y aspectos negativos en la organización de la Universidad (administrativo y logístico), son mayores sus deseos de culminar con éxito su carrera profesional. Para ellos es importante el aprendizaje autónomo y lo consideran principal responsable del éxito académico. También consideran que esta modalidad permite fomentar factores resilientes como la introspección y la responsabilidad. 
Peralta S., (2006), ${ }^{40}$ concluyó en la investigación realizada en la Universidad de Sucre, que la iniciativa es el único factor, entre los demás factores resilientes que es determinante en el rendimiento académico alto o bajo de los estudiantes de esa Universidad, estos resultados se corroboraron en la presente investigación y se encontró que los estudiantes con iniciativa son personas autónomas, con capacidad de decisión y críticos frente a su propia vida.

Estos resultados demuestran que el factor de la introspección es el que más repercute en el éxito académico de los estudiantes de la UNAD, ya que éste les permite reconocer sus falencias y buscar alternativas para superarlas. Esto se analizó con base en las respuestas dadas por los estudiantes al cuestionario CREU y a la entrevista semiestructurada.

El Segundo factor encontrado es el factor de la iniciativa definida por Werner (1982) y Wolin y Wolin (1993), citado por peralta S. (2006) como la capacidad de tener comportamientos dirigidos hacia una meta, es decir, que planean con anterioridad sus acciones y fijan fechas concretas para el cumplimiento de objetivos, ${ }^{41}$ los estudiantes de la UNAD manifiestan ser personas perseverantes que luchan por alcanzar sus objetivos sin dejarse vencer por los obstáculos que se les presentan durante el proceso académico.

Por otra parte, en una investigación realizada en alianza con las universidades HUJ (Universidad Hebrea de Jerusalén) y la UNAD, en desarrollo del software Dígalo, titulada Argumentación en ambientes digitales de la educación; una experiencia con reintegrados a la vida civil, uno de los principales hallazgos fue que el trabajo en equipo es una actitud positiva que permite alcanzar metas y objetivos comunes debido a que fomenta lazos de amistad y compañerismo, repercute en la autoestima de los participantes e influye en la confianza y la tolerancia para con los demás. En esta investigación se ha comprobado que los grupos colaborativos implementados en la UNAD, fomentan de manera significativa el factor resiliente de la interacción desarrollando capacidades, habilidades y destrezas.

Este factor amplia las redes sociales y fomenta los pilares de la resiliencia comunitaria planteada por Néstor Suárez Ojeda (2001), como la autoestima colectiva, identidad cultural, honestidad estatal, solidaridad, humor social y liderazgo comunitario. Cuando los individuos están en interacción con otros, se promueve el aprendizaje social, permitiendo así aprender de las fortalezas de los demás y potencializar las propias, generando una autoestima colectiva gracias al complemento de los integrantes del grupo.

\section{CONCLUSIONES}

Los resultados de esta investigación permitieron identificar los factores resilientes asociados con el éxito académico y la permanencia de un grupo de estudiantes de la UNAD, y podrán contribuir en próximas investigaciones y/o acciones de intervención,

40 Peralta S., 2006, Factores Resilientes Asociados al Rendimiento Académico de la Universidad de Sucre.

41 Citados por Peralta S., 2006, en su investigación, Factores Resilientes Asociaos al Rendimiento Académico en la Universidad de Sucre. 
con el objeto de potencializar dichos factores. Los factores que más se presentan en los estudiantes de la UNAD son: primero, el factor de la introspección es el que más repercute en el éxito académico, seguido por la interacción, que se fomenta con el trabajo en equipo desarrollando capacidades, habilidades y destrezas, en los estudiantes y, por último, la iniciativa definida por Werner (1982) y Wolin y Wolin (1993), citado por peralta S., (2006) como la capacidad de tener comportamientos dirigidos hacia una meta.

Los estudiantes que reconocen sus debilidades y falencias y buscan la forma corregirlas por lo general poseen el factor de la introspección que contribuye al éxito académico.

Los grupos colaborativos fomentan la resiliencia comunitaria ya que al interactuar con otros promueven el aprendizaje social, potencializando así las fortalezas propias y la de los demás.

En los países subdesarrollados y con muchos problemas sociales como Colombia la educación a distancia es una alternativa viable para las personas que no cuentan con los recursos o el tiempo para acceder a un aula educativa de manera permanente y presencial. De esta manera adquieren un compromiso personal con su aprendizaje y con la adquisición de conocimientos que le llevarán a mejorar su calidad de vida.

\section{REfERENCIAS BIBLIOGRÁfICAS}

ARGUEDAS L. (2.004). Potenciación de la resiliencia: una alternativa para la permanencia y el éxito académico de los estudiantes de la UNED. Costa Rica.

CARDONA, P \& OTROS. (2008) Módulo aspectos psicosociales de la resiliencia. Universidad Nacional Abierta y a Distancia UNAD.

GARMEZY, N (1994). Rflections and commentary on risk, resilience, and development, In R.J. Haggerty, L.R. Sherrod, N. Interventions (pp.1-18).

LANDAZÁBAL \& Et al. (2007) Una luz que Brilla, La resiliencia. Editorial UNAD.

LÓAIZA, F., RAMÍREZ, H.., LINDO, M. (2001)La universidad a distancia y la universidad virtual: "El modelo pedagógico" Revista de Ciencia Humanas. No. 28.

LUCIANO DI GRESIA Y ALBERTO PORTO (2001), "Rendimiento de Estudiantes Universitarios y sus Determinantes".

MALAGÓN L, (2.003). La investigación en educación abierta y a distancia.

PERALTAS; RAMÍREZA; CASTAÑO H. (2002), Factores resilientes asociados al rendimiento académico en estudiantes pertenecientes a la universidad de sucre, Colombia. 
ROJAS, J, (2008) La educación a distancia en Colombia: una historia que vale la pena contar. Programa Formador de formadores UNAD.

ROJAS, J. (2003) Seminario de Inducción Unadista, SIU; p. 110-111. Universidad Nacional Abierta y a Distancia.

SILVA D, GLAZ C, 2000, Análisis en Resiliencia y aprendizaje en sectores populares.

SILVA, D, GLAZ C, (2000). Resiliencia y aprendizaje en sectores populares. Argentina.

TOMADO DE BRENES ESPINOSA, FERNANDO. Principios y fundamentos para una teoría de la educación a distancia. Centro de capacitación y estudios en educación a distancia. Recuperado el 22, marzo, 2008 en: http://www.csuca.edu.gt/Sistemas/SICAR/ sep_files/aplicacion_alumnos/contenidos/2_model13.htm.

TOMADO DE ADAMES, PEDRO ET AL (2002). Teorías de la educación a distancia. Recuperado el 15, marzo, 2008) en: http://virtual.ucla.edu.ve/ciencias/teorias/archivos/I. ppt\#292.

TOMADO DE GALARZA PÉREZ, LUÍS. El estudio independiente en una visión sistémica de la educación a distancia. Recuperado el 21, marzo, 2008 en: http://www.mse.buap. $\mathrm{mx} / \mathrm{pdhd} /$ cursos/EstudioIndependiente/contenido/BLIBLIOGRAFIA_archivos/Tema_1/ Luis_Galarza_El_estud_independ.pdf.

TOMADO DE BRENES ESPINOSA, FERNANDO. Principios y fundamentos para una teoría de la educación a distancia. Centro de capacitación y estudios en educación a distancia. Recuperado el 22, marzo, 2008 en: http://www.csuca.edu.gt/Sistemas/SICAR/sep_files/ aplicacion_alumnos/contenidos/2_model13.htm http://cmapspublic.ihmc.us/servlet/SBRea dResourceServlet?rid=1174976814031_733554291_7595\&partName=htmltext.

TOMADO DE ADAMES, PEDRO ET AL (2002). Teorías de la educación a distancia. Recuperado el 15, marzo, 2008) en: http://virtual.ucla.edu.ve/ciencias/teorias/archivos/I. ppt\#292,2, Contenido.

TOMADO DE ADAMES, PEDRO ET AL (2002). Teorías de la educación a distancia. Recuperado el 15, marzo, 2008) en: http://virtual.ucla.edu.ve/ciencias/teorias/archivos/I. ppt\#292,2,Contenido.

TOMADO DE BRENES ESPINOSA, FERNANDO. Principios y fundamentos para una teoría de la educación a distancia. Centro de capacitación y estudios en educación a distancia. Recuperado el 22, marzo, 2008 en: http://www.csuca.edu.gt/Sistemas/SICAR/ sep_files/aplicacion_alumnos/contenidos/2_model13.htm. 
TOMADO DE GALARZA PÉREZ, LUÍS. El estudio independiente en una visión sistémica de la educación a distancia. Recuperado el 21, marzo, 2008.

TOMADO DE GALARZA PÉREZ, LUÍS. El estudio independiente en una visión sistémica de la educación a distancia. Recuperado el 21, marzo, 2008.

TOMADO DE LA DIPLOMATURA FORMADOR DE FORMADORES, curso Acompañamiento Tutorial en EAD y AVA.

SCHIMID A. (2.000). En defensa de la educación a distancia, los desafíos de la capacitación docente, articulo investigativo en las provincias de Buenos Aires (argentina), San Juan, Catamarca, Río Negro y Chubut.

MELÉNDEZ, J. (1993). La efectividad de la educación a distancia como metodología en el desarrollo de destrezas de pensamiento. En www.ateneonline.net/datos/105_01_Schmid_ Ana.pdf. 